\title{
Radiology teaching for junior doctors: their expectations, preferences and suggestions for improvement
}

\author{
Christiane M. Nyhsen • Cathy Lawson • Jim Higginson
}

Received: 1 August 2010/Revised: 12 October 2010 /Accepted: 29 October 2010 /Published online: 29 January 2011

(C) European Society of Radiology 2011

\begin{abstract}
Objectives To evaluate radiology teaching offered to junior doctors on general medical/surgical rotations, their preferences regarding teaching methods and self-directed learning tools, and their suggestions for improvement.

Methods An online questionnaire involving all foundation programme doctors at Sunderland Royal Hospital was carried out. Quantitative analysis of preferences and free text feedback were used.

Results There was a response rate of $88 / 90$ doctors (98\%). The radiology teaching received was mostly informal. Most junior doctors felt that their medical school radiology teaching had been inadequate. The preferred teaching techniques were interactive case-based and system-based discussions. Textbooks and journal articles were not as popular as self-directed learning tools. Online learning material was used quite frequently, with general web content being more popular. Eighty-seven percent cited their motivation for studying radiology as "to become a better doctor". More guidance from radiologists was desired, particularly regarding the choice of examinations and discussion of cases. Twenty-two percent of doctors were considering radiology as a career.

Conclusions Interactive elements in radiology teaching are important. Online electronic teaching modules can be
\end{abstract}

C. M. Nyhsen $(\bowtie)$

Consultant Radiologist, Department of Radiology, Sunderland

Royal Hospital,

Kayll Road,

Sunderland SR4 7TP, UK

e-mail: Christiane.Nyhsen@chs.northy.nhs.uk

C. Lawson · J. Higginson

Foundation Year 2 Doctor, Sunderland Royal Hospital,

Kayll Road,

Sunderland SR4 7TP, UK integrated into the teaching curriculum, but they must be of high quality to be acceptable and face-to-face interaction is still important. Junior doctors would like more guidance from radiologists.

Keywords Hospitals · Teaching · Teaching methods · Radiology

\section{Introduction}

Providing radiology teaching for junior doctors (nonradiologists) and medical students is an important but often neglected task. Many images are reviewed by junior staff, particularly out of hours, before being formally reported by a radiologist or seen by an experienced consultant on the ward round. It is, therefore, in the best interests of patients to have well-educated junior staff reviewing images, so that critical findings are not missed and obvious abnormalities not misinterpreted.

According to the literature, there has been little research regarding the expectations of junior doctors with respect to radiology teaching, their motivation and their preferred teaching methods. We have therefore conducted a survey of our junior doctors to explore this area.

\section{Materials and methods}

Data were collected in late 2009/early 2010 (approximately halfway through the year of training) using an online questionnaire (based in Google Documents) aimed at foundation year-one and -two trainees working in the Sunderland Royal Hospital, UK. These are doctors in their first two years of training following university graduation, 
who complete mixed surgical/medical rotations before entering specialty training.

Ninety doctors were included. The initially poor response rate to the emailed online questionnaire was improved considerably by telephoning all doctors individually and conducting the questionnaire by phone where indicated. A final response rate of 88/90 doctors (98\%) was thereby achieved.

The questionnaire included a range of subjective questions about the perceived quality of training or effectiveness of teaching methods. Hereby score 1 was named "poor" or "not effective" and a score 5 equalled "excellent" or "very effective".

\section{Results}

\section{Demographics}

Fifty-nine percent of the responding foundation-year doctors were female. Most doctors were aged between 22 and 27 (85\%); 5\% were aged between 28 and 31, and $10 \%$ were over 31 years old.

\section{Overall impressions}

Forty-eight percent of respondents reported that, in the preceding months, they had had weekly formal as well as informal radiology teaching. Twenty-nine percent recalled monthly teaching, $7 \%$ reported daily exposure, whereas $11 \%$ said that they had no experience of radiology teaching at all (4\% reported annual exposure and $1 \%$ did not complete the question).

Of the radiology teaching received, most teaching was informal; this was rated positively for the most part. Formal teaching, by contrast, attracted mixed ratings.

When asked whether-in retrospect-the radiology teaching provided as a medical student had been adequate, $64 \%$ of doctors stated that they had too little exposure to radiology. $31 \%$ said that exposure to radiology was "about right" and only $5 \%$ stated that it was "too much".

Teaching methods and self-directed learning

Interactive case-based discussion was clearly the favourite teaching technique, followed by interactive system-based discussions (see Fig. 1).

PowerPoint-aided lectures mostly attracted ratings from average to good, whereas examination-style viva sessions received mixed reviews. Interestingly, the evaluation of self-directed e-learning modules showed a graph looking very similar to a normal distribution (see Fig. 2).

Original research articles, journal review articles and radiology textbooks were reported as rarely used. Other more general textbooks were a little more popular.
Dedicated online learning material was moderately to regularly accessed but other web resources, such as Google and Wikipedia, were used much more widely (see Fig. 3).

Expectations of teaching outcomes

Respondents were asked which radiological investigations they would expect to be able to interpret confidently following appropriate radiology teaching at medical school. Most doctors mentioned chest and abdominal as well as other radiographs first, followed by head computed tomography (CT; 56\%) and other CT imaging (27\%). A significant minority also stated that they would like to be able to interpret magnetic resonance imaging (MRI), ultrasound images and some other investigations (see Fig. 4).

\section{Motivation}

Eighty-seven percent of respondents cited "becoming a better doctor" as their motivation for studying radiology (see Fig. 5). Ten percent reported a personal interest, and $1 \%$ considered a potential career in radiology as the major motivating factor. Only a negligible minority thought about "passing the next examination" or "looking good on ward rounds" ( $1 \%$ each).

When asked whether or not the junior doctors would consider radiology as a career option, $22 \%$ replied yes.

General comments in the free text sections of the questionnaire included junior doctors often feeling lost in the radiology department, in particular when requesting urgent investigations. Many stated that they wanted to learn more about radiological examination techniques, preparation of patients and suitability of patients for certain examinations. They also often felt unsure about the choice of the correct investigation.

\section{Discussion}

From the data we extracted it is clear that junior doctors value interactive sessions most highly. Similar findings were reported by Malek et al. [1], who concluded that "interactivity was highly valued by students". Sessions with interactive elements also appear to achieve better results, which may be due to the "stimulation of critical thinking" [2] and possibly better concentration in groups using interactive elements [1].

The junior doctors we questioned preferred case-based interactive sessions to system-based teaching sessions. Several studies have already examined case-based teaching in radiology and found that this may have a beneficial effect on students' problem-solving ability [1]. Chew [3] described case conferences, which proved very popular amongst radiology residents. Unfortunately there does not appear to be a direct comparison study to evaluate whether 
Fig. 1 Rating of interactive case based teaching
How do you rate interactive case based discussion teaching?

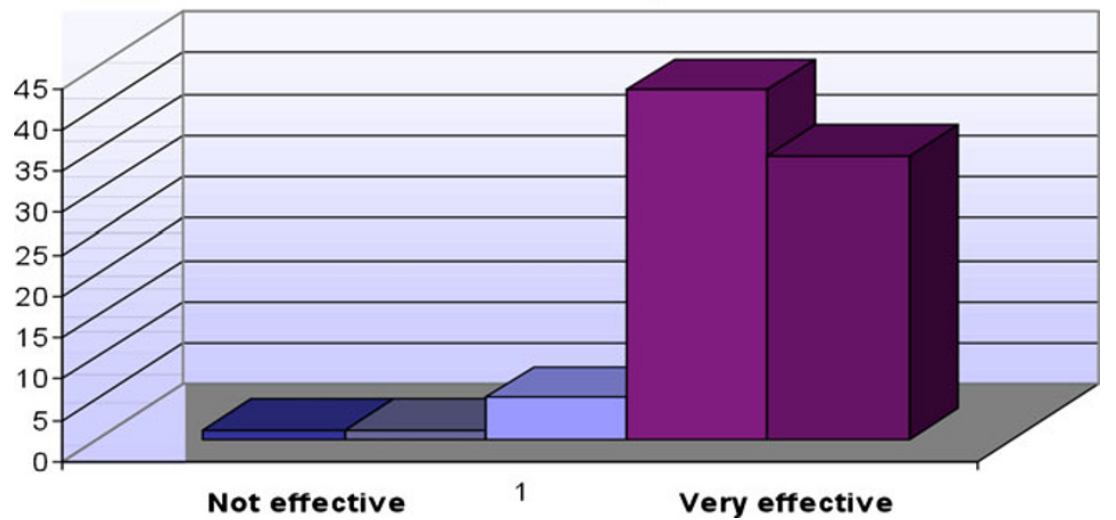

case-based teaching overall is in fact more effective (i.e. yielding better results) than system-based teaching.

The preference of case-based studies, as found in our study, can also be seen in the context of the doctors' primary motivation when studying radiology, which most respondents stated was "to become a better doctor". Using real cases in teaching sessions and then extracting learning points that are closest to daily medical practice is the most popular teaching method and one would also assume the most effective way to retain information regarded by the doctors as relevant to their jobs.

This phenomenon is obviously not new but has been described in many learning theories. With this study, however, we can confirm that it is just as relevant to young doctors today as it was to students a few decades ago.

Contrary to the consistently preferred teaching methods, the use of self-directed learning resources has seen a shift in preferences that reflects the changing use of technology and media in general. As Kitchin and Applegate [4] have described, traditional textbooks are being dropped in favour of online resources and short case review books. Our study also found that radiology textbooks, review articles and original research were rarely used by young doctors.
They may be unpopular as they are deemed too subspecialist, or it may simply be the length and depths of topics explored. Generalist textbooks were consulted more frequently, possibly as the content is broader and pathological conditions are not discussed in as much detail.

In summary, our results seem to suggest that younger doctors prefer more "brief and digested" forms of information, and learning styles that are reflective of encounters in daily medical practice.

Analysis of the more traditional styles of teaching showed that PowerPoint-aided lectures were preferred to examinationstyle viva sessions, or "grilling". It would be interesting to see further research to establish whether the unpopularity of these examination-style teaching sessions is related to the discomfort felt by students during the sessions.

Changing the format of case-based discussions as described by Chew [3] and allowing preparation of cases beforehand certainly seems to be better received. In addition, the author reports that trainees appeared more engaged and the number of teaching cases could be increased, maximising the teacher's efforts-although it can be argued of course that more preparation time may initially be needed.
Fig. 2 Rating of e-learning modules

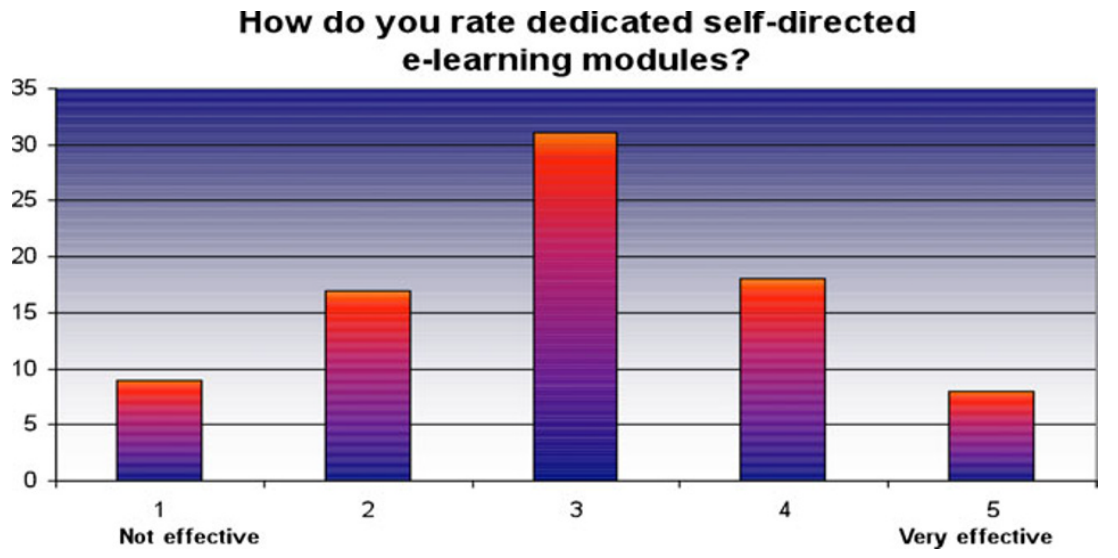


General web content use for self-directed learning

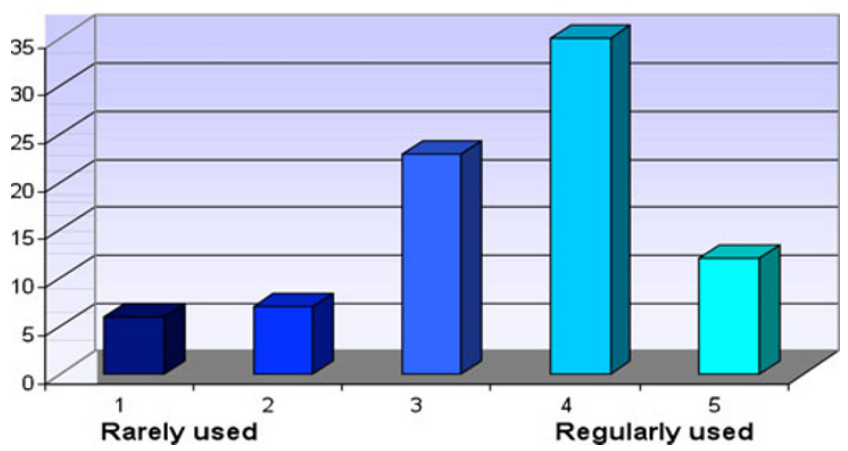

Fig. 3 Use of the internet as a source for self-directed learning

Interestingly, online teaching resources and dedicated e-learning modules were not highly valued by all doctors, whereas most professed regular use of general web resources. This raises a few points:

First, not all specialist online learning content is deemed of high quality and possibly of adequate level for the respective trainees.

Second, there may be "insufficient maturity of current e-learning software" [5] and not all software used allows interactive elements. These interactive elements have been shown to enhance learning (see above), and so perhaps their absence discourages use of the respective resources.

Third, there may be a "lack of incentives and rewards" for doctors using the resources [5].

Fourth, many doctors still prefer face-to-face teaching. Rowell et al. [6] reported that although web-based resources were deemed reliable and the web was regularly used for information gathering, responders still preferred hospital-run CME courses and assessed them as "more effective and efficient". Lieberman et al. [7] also found that not only "more students said that they liked tutorial learning" in comparison with computer-assisted instruction, but they also found that test results were marginally but significantly better after "human teaching". The latter was attributed to increased interaction, concentration and the obligation to complete the entire teaching session without opportunities to "skip" part of the online teaching.
So some limitations of online teaching resources have to be accepted. Although the younger generation can be expected to be more open to online resources and to be better able to cope with the technical elements, the motivation and stimulation will not be the same as having a session with an inspiring teacher. Regarding the motivation, other incentives can be thought of, like certificates for successful completion of online tutorial and tests, etc. Whilst a computer can arguably transfer information as competently as any teacher (and possibly more consistently), it cannot encourage an individual to care about that information like a real person. This is not to say that online teaching modules cannot be integrated into the training curriculum and more universities and other sites now offer access to their online teaching facilities $[5,8]$.

Electronic tutorials may be a good means of ensuring that basic knowledge is acquired before a small group tutorial, for example, where pathological conditions can then be discussed in greater breadth and depth, and questions can be answered. This would optimise teacher's time spent with students and (once established) it may be more cost effective (i.e. saving money to pay a teacher to deliver the training), an important factor in the current financial climate.

Ochoa and Wludyka [2] state that web-based technology is still "underused for teaching purposes" and several other authors also suggest the integration of online resources side by side with more traditional delivery of the curriculum [911]. Our hospital is aiming to integrate online tutorials into the curriculum in the coming academic year.

A further valuable point extracted from our survey is that almost two-thirds of doctors reported that they did not receive enough radiology teaching as medical students, and thus felt ill-prepared for their first jobs post-graduation. This is a point that is to be taken seriously.

Radiologists should take an interest in teaching medical students, as only then can this shortfall be addressed. Raising awareness of and interest in radiology early in students' careers should also have the beneficial effect of positively selecting high-quality students and future doctors to radiology training schemes. Let the best students and young doctors have a professional insight into what we do,
Fig. 4 Non-radiology trainees' espectations regarding the outcome of radiology training

\section{With appropriate teaching, which investigations would you expect to be} able to interpret with confidence

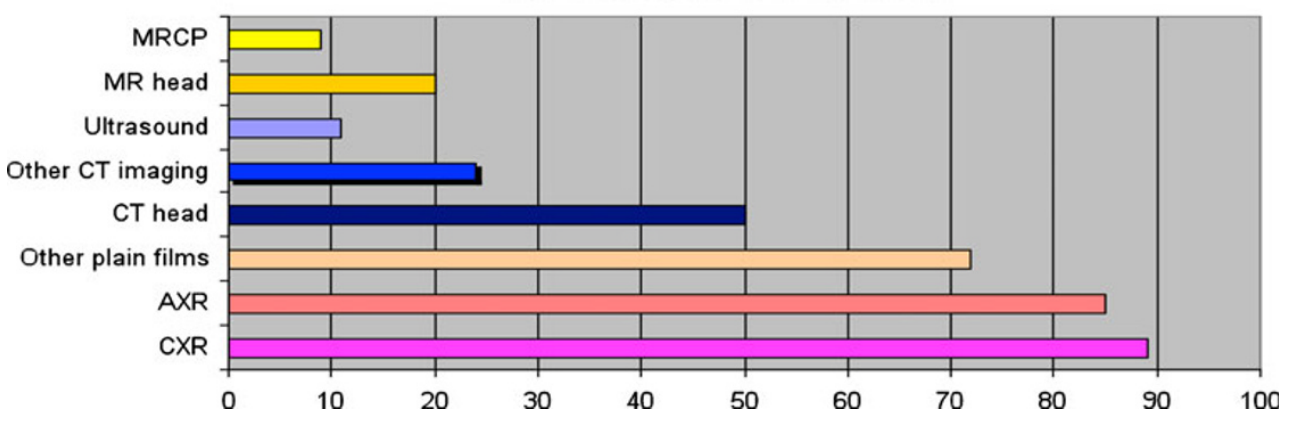


Fig. 5 Doctors' motivation to study radiology

\section{Primary motivation of questioned doctors when studying radiology}

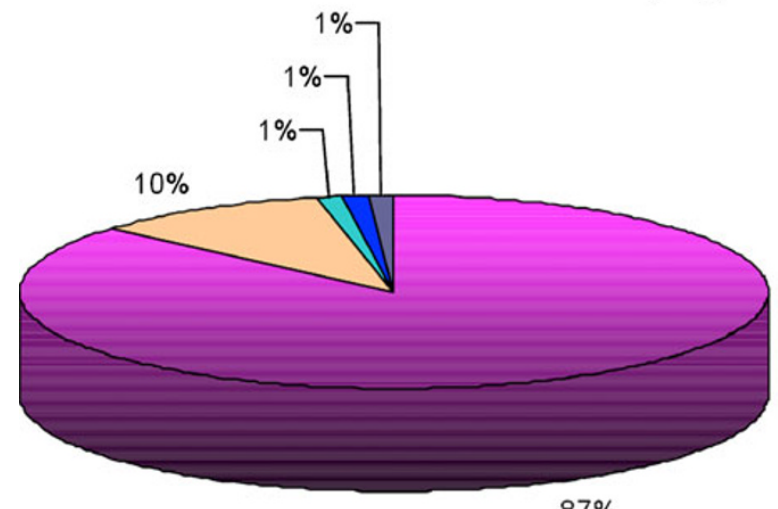

$87 \%$ $\square$ Becoming a better doctor

$\square$ Personal interest

$\square$ Looking good on wardrounds

- Considering a career in radiology

$\square$ Passing the next exam then the quality of applicants for radiology training will improve.

Studies by Gunderman et al. [12] and Branstetter et al. $[13,14]$ confirm the thesis that "good medical student teaching pays important dividends". This applies to the first couple of years in medical school (where radiology can be integrated in anatomy teaching) as well as later clinical years.

The perception of radiology departments has improved according to the answers provided regarding whether or not doctors would consider radiology as a career option. A surprising $22 \%$ answered this positively, which means that we have hopefully left behind the image of being "grumpy creatures sitting in the dark" who "never have any patient contact". The UK Medical Careers Research Group supports our encouraging findings [15].

Multi-disciplinary team meetings may have helped to highlight the importance of radiology. Young doctors now see that radiologists provide significant input in patient management. Furthermore, picture archiving and communication systems (PACS) now allow a new transparency of the radiology department with the ability to view the full range of our diagnostic and therapeutic procedures.

The advent of PACS may also have contributed to our observation that junior doctors were interested in learning to interpret more complex images such as $\mathrm{CT}$ and MRI. When asked about the young doctors' expectations, and which investigations they thought they should be able to interpret with confidence following appropriate teaching, most cited plain films, as expected. However, over half of doctors also stated that they would expect to be able to interpret head CT, and just over a quarter would like to be able to interpret other $\mathrm{CT}$ investigations. A significant minority also wanted to know more about interpretation of MRI and ultrasound images.

Teaching all these investigations to a standard where the trainees could "interpret the images with confidence" remains well beyond any reasonable radiology allocation within a medical student's or junior doctor's curriculum. However, seeing that the interest is there, some basic training could be considered, in particular as the next generations of doctors will inevitably look at images on PACS and try to interpret them.

Being able to recognise some major findings (like an intracranial haemorrhage or metastasis) may be helpful, in particular if there should be a delay in getting the images reported by a radiologist for whatever reason. It has to be stressed, however, that teaching needs to ensure that trainees are able to recognise their own limitations and learn to appreciate the complexity of examinations. They need to always seek the specialist radiology report before taking action on presumed findings and thereby potentially missing important concurrent pathological conditions.

The free text section at the end of the questionnaire drew a wide variety of responses. "More guidance from radiologists" was the main request, again perhaps a reflection of the paucity of teaching at undergraduate level. Junior doctors generally want to improve their skills and do the best for their patients, but are unsure how to speak to a radiologist. They are often anxious because of uncertainty about which information is essential, which examinations are appropriate, and what patient preparation is necessary. It seems that in this respect, referring consultants from other specialties does not always offer enough insight into why they are planning a certain investigation and leave it to the junior doctors to come down to radiology to request them urgently. They can subsequently feel a bit "lost" in our department, and perhaps rather afraid of getting it wrong and being "told off" by a radiologist.

As a result of this survey, we are now offering to the foundation doctors starting in our hospital a new introductory session with a consultant radiologist. This covers a general introduction to the radiology department as well as practical hints on how to request urgent imaging in particular, what information is needed, what patient preparation is useful and the common pitfalls to avoid. 
Acknowledgements We would like to thank Mrs Catherine Fisher, Chief librarian at the Sunderland Royal Hospital, and her colleagues for their outstanding support.

\section{References}

1. Maleck M, Fischer MR, Kammer B et al (2001) Do computers teach better? A media comparison study for case-based teaching in radiology. Radiographics 21(4):1025-1032

2. Ochoa JG, Wludyka P (2008) Randomized comparison between traditional and traditional plus interactive Web-based methods for teaching seizure disorders. Teach Learn Med 20(2):114-117

3. Chew FS (2001) The case-based radiology teaching conference for residents: beneficial effect of previewing cases and using answer sheets. Acad Radiol 8(10):993-997

4. Kitchin DR, Applegate KE (2007) Learning radiology: a survey investigating radiology resident use of textbooks, journals, and the internet. Acad Radiol 14:1113-1120

5. Sparacia G, Cannizzaro F, D'Alessandro DM et al (2007) Initial experiences in radiology e-learning. Radiographics 27(2):573-581

6. Rowell MR, Johnson PT, Fishman EK (2007) Radiology education in 2005: world wide web practice patterns, perceptions, and preferences of radiologists. Radiographics 27(2):563-571

7. Lieberman G, Abramson R, Volkan K et al (2002) Tutor versus computer: a prospective comparison of interactive tutorial and computer-assisted instruction in radiology education. Acad Radiol 9:40-49

8. Scarsbrook AF, Graham RN, Perriss RW (2006) Radiology education: a glimpse into the future. Clin Radiol 61(8):640-648

9. Howlett D, Vincent T, Watson G et al (2009) Blending online techniques with traditional face to face teaching methods to deliver final year undergraduate radiology learning content. Eur J Radiol. doi:10.1016/j.ejrad.2009.07.028

10. Armstrong P, Elliott T, Ronald J, Paterson B (2009) Comparison of traditional and interactive teaching methods in a UK emergency department. Eur J Emerg Med 16(6):327-329

11. Kourdioukova EV, Valcke M, Verstraete KL (2010) The perceived long-term impact of the radiological curriculum innovation in the medical doctors training at Ghent University. Eur J Radiol. doi:10.1016/j.ejrad.2010.06.022

12. Gunderman RB, Alexander S, Jackson VP et al (2000) The value of good medical student teaching: increasing the number of radiology residency applicants. Acad Radiol 7(11):960-964

13. Branstetter BF 4th, Faix LE, Humphrey AL et al (2007) Preclinical medical student training in radiology: the effect of early exposure. Am J Roentgenol 188(1):W9-W14

14. Branstetter BF 4th, Humphrey AL, Schumann JB (2008) The long-term impact of preclinical education on medical students' opinions about radiology. Acad Radiol 15(10):1331-1339

15. Turner G, Lambert TW, Goldacre MJ (2006) Career choices for radiology: national surveys of graduates of 1974-2002 from UK medical schools. Clin Radiol 61(12):1047-1054 